\title{
Pyruvate dehydrogenase kinase inhibition: Reversing the Warburg effect in cancer therapy
}

\author{
Hayden Bell, Edward Parkin
}

Division of Biomedical and Life Sciences, Faculty of Health and Medicine, Lancaster University, Lancaster, United Kingdom

Received February 02, 2016; Revised June 06, 2016; Accepted June 09, 2016; Published Online June 23, 2016

\section{Review Article}

\begin{abstract}
The poor efficacy of many cancer chemotherapeutics, which are often non-selective and highly toxic, is attributable to the remarkable heterogeneity and adaptability of cancer cells. The Warburg effect describes the up regulation of glycolysis as the main source of adenosine 5'-triphosphate in cancer cells, even under normoxic conditions, and is a unique metabolic phenotype of cancer cells. Mitochondrial suppression is also observed which may be implicated in apoptotic suppression and increased funneling of respiratory substrates to anabolic processes, conferring a survival advantage. The mitochondrial pyruvate dehydrogenase complex is subject to meticulous regulation, chiefly by pyruvate dehydrogenase kinase. At the interface between glycolysis and the tricarboxylic acid cycle, the pyruvate dehydrogenase complex functions as a metabolic gatekeeper in determining the fate of glucose, making pyruvate dehydrogenase kinase an attractive candidate in a bid to reverse the Warburg effect in cancer cells. The small pyruvate dehydrogenase kinase inhibitor dichloroacetate has, historically, been used in conditions associated with lactic acidosis but has since gained substantial interest as a potential cancer chemotherapeutic. This review considers the Warburg effect as a unique phenotype of cancer cells in-line with the history of and current approaches to cancer therapies based on pyruvate dehydrogenase kinase inhibition with particular reference to dichloroacetate and its derivatives.
\end{abstract}

Keywords: Pyruvate Dehydrogenase Kinase; Warburg; Dichloroacetate; Cancer

\section{Introduction}

Energy production in cells can occur relatively inefficiently via glycolytic conversion of glucose to pyruvate in the cytosol. Alternatively, much more efficient energy production can arise via the conversion of pyruvate to acetyl-Coenzyme A (acetyl-CoA) and the subsequent tricarboxylic acid (TCA) cycle in the mitochondria. Cancer cells tend to utilize the former pathway more than normal cells. This shift of energy production away from the mitochondria to the glycolytic pathway is referred to as the 'Warburg effect' after its proposer Otto Heinrich Warburg. ${ }^{1}$ Reversing the Warburg effect, thereby shifting energy production back to the mitochondria and potentially reinstating mitochondrial apoptotic pathways, has become an attractive potential treatment for cancer in recent years. A particularly attractive therapeutic target in this respect is the enzymatic activity of the mitochondrial pyruvate dehydrogenase complex (PDC) which sits at the interface between glycolysis and the TCA and, therefore, regulates the metabolic fate of glucose down one or the other pathway.

PDC catalyzes the irreversible oxidative decarboxylation of pyruvate to acetyl-CoA via a sequential mechanism (Figure 1). This acetyl-CoA, along with the acetyl-CoA from fatty acid $\beta$-oxidation, enters the TCA cycle to produce the electron donors $\mathrm{NADH}$ and $\mathrm{FADH}_{2}$. The electron transport to molecular oxygen via respiratory chain complexes then leads to the formation of a proton gradient across the inner mitochondrial membrane. The electrochemical potential energy within this proton gradient is utilized to drive the formation of ATP via mitochondrial ATP synthetase.

The PDC contains multiple copies of three different catalytic components. The E1, pyruvate dehydrogenase, component (pink) catalyzes the decarboxylation of pyruvate to hydroxyethyl-TPP and, subsequently, the reductive acetylation of lipoyl moieties on the E2,

Corresponding author: Edward Parkin; Division of Biomedical and Life Sciences, Faculty of Health and Medicine, Lancaster University, Lancaster, United Kingdom.

Cite this article as: Bell H, Parkin E. Pyruvate dehydrogenase kinase inhibition: Reversing the Warburg effect in cancer therapy. Int J Cancer Ther Oncol. 2016; 4(2):4215. DOI: 10.14319/ijcto.42.15 
dihydrolipoamide acetyltransferase, component (blue). The E2 component then transfers these acetyl groups to CoA to form acetyl-CoA. The E3, dihydrolipoamide dehydrogenase, component (green) utilizes a FAD prosthetic group to catalyze the oxidation of the reduced lipoyl moieties on the E2 component with the concomitant reduction of $\mathrm{NAD}^{+}$.

Situated within the mitochondrial matrix, PDC is a highly integrated multimeric enzyme complex containing multiple copies of three different catalytic molecules that, collectively, catalyze the sequential oxidative decarboxylation of pyruvate (Figure 1). ${ }^{4}$ First, the pyruvate dehydrogenase (E1) component catalyzes the decarboxylation of pyruvate to hydroxyethyl-thiamine pyrophosphate (hydroxyethyl-TPP) followed by the reductive acetylation of lipoyl moieties on the dihydrolipoamide acetyltransferase (E2) component. The latter component then transfers the acetyl moieties to CoA to form acetyl-CoA. Finally, the dihydrolipoamide dehydrogenase (E3) component, utilizing its FAD prosthetic group, catalyzes the oxidation of the reduced lipoyl moieties on the E2 component with the concomitant reduction of $\mathrm{NAD}^{+}$.

\section{Pyruvate dehydrogenase complex E1 $\alpha$ regulation}

At such a crucial and central position within normal cellular metabolism, the catalytic activity of PDC is subject to meticulous regulation with many mechanisms acting in concert including: allostery; reversible phosphorylation; reversible acetylation; and reversible succinylation (Figure 2). These post-translational modifications are, in turn, themselves regulated contributing to the elaborate regulation of carbon flux through the PDC.

The principal regulation of mammalian PDC is achieved through the site-specific, inhibitory phosphorylation of the $\alpha$ subunits of the heterotetrameric $\left(\alpha_{2} \beta_{2}\right)$ E1 component at three different sites - Ser293 (site 1), Ser300 (site 2), and Ser232 (site 3). ${ }^{5}$ The presence of three different phosphorylation sites confers a level of complexity to PDC regulation and allows fine-tuning to metabolic requirements achieved through the activities of pyruvate dehydrogenase kinase (PDK) and pyruvate dehydrogenase phosphatase (PDP). However, it has been shown, through site-directed mutagenesis, that phosphorylation at any one site of the E1 component leads to inhibition of the catalytic component. ${ }^{6}$ Site-specific phosphorylation of E1 $\alpha$ results in E1 inactivation via different mechanisms; for example, phosphorylation of site 1 prevents steric interaction of reaction substrates with the $\mathrm{E} 1$ active site, whereas site 3 phosphorylation affects co-enzyme, TPP, binding to E1.7, 8 Additionally, phosphorylation of E1 $\alpha$ at Tyr301 blocks binding of substrate (pyruvate), thereby inhibiting the catalytic component in a similar manner to Ser293 phosphorylation. ${ }^{9}$

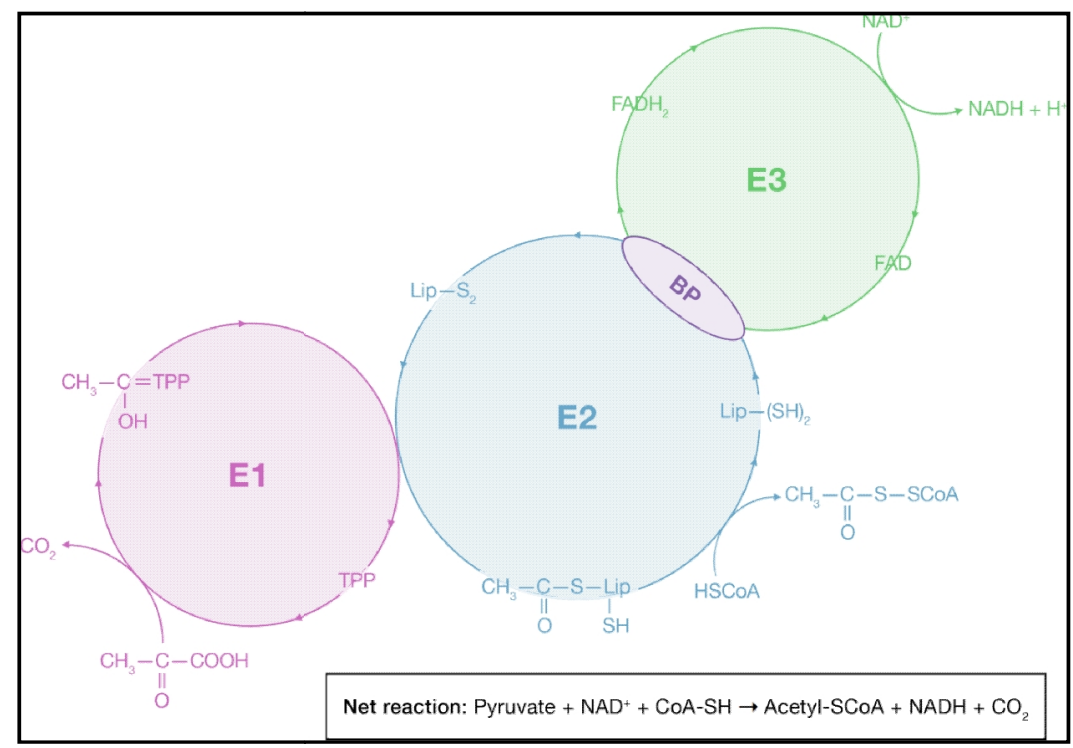

Figure 1: The catalytic mechanism of the pyruvate dehydrogenase complex (adapted ${ }^{2,3}$ ). The PDC contains multiple copies of three different catalytic components. The E1, pyruvate dehydrogenase, component (pink) catalyzes the decarboxylation of pyruvate to hydroxyethyl-TPP and, subsequently, the reductive acetylation of lipoyl moieties on the E2, dihydrolipoamide acetyltransferase, component (blue). The E2 component then transfers these acetyl groups to CoA to form acetyl-CoA. The E3, dihydrolipoamide dehydrogenase, component (green) utilizes a FAD prosthetic group to catalyze the oxidation of the reduced lipoyl moieties on the E2 component with the concomitant reduction of NAD+. 


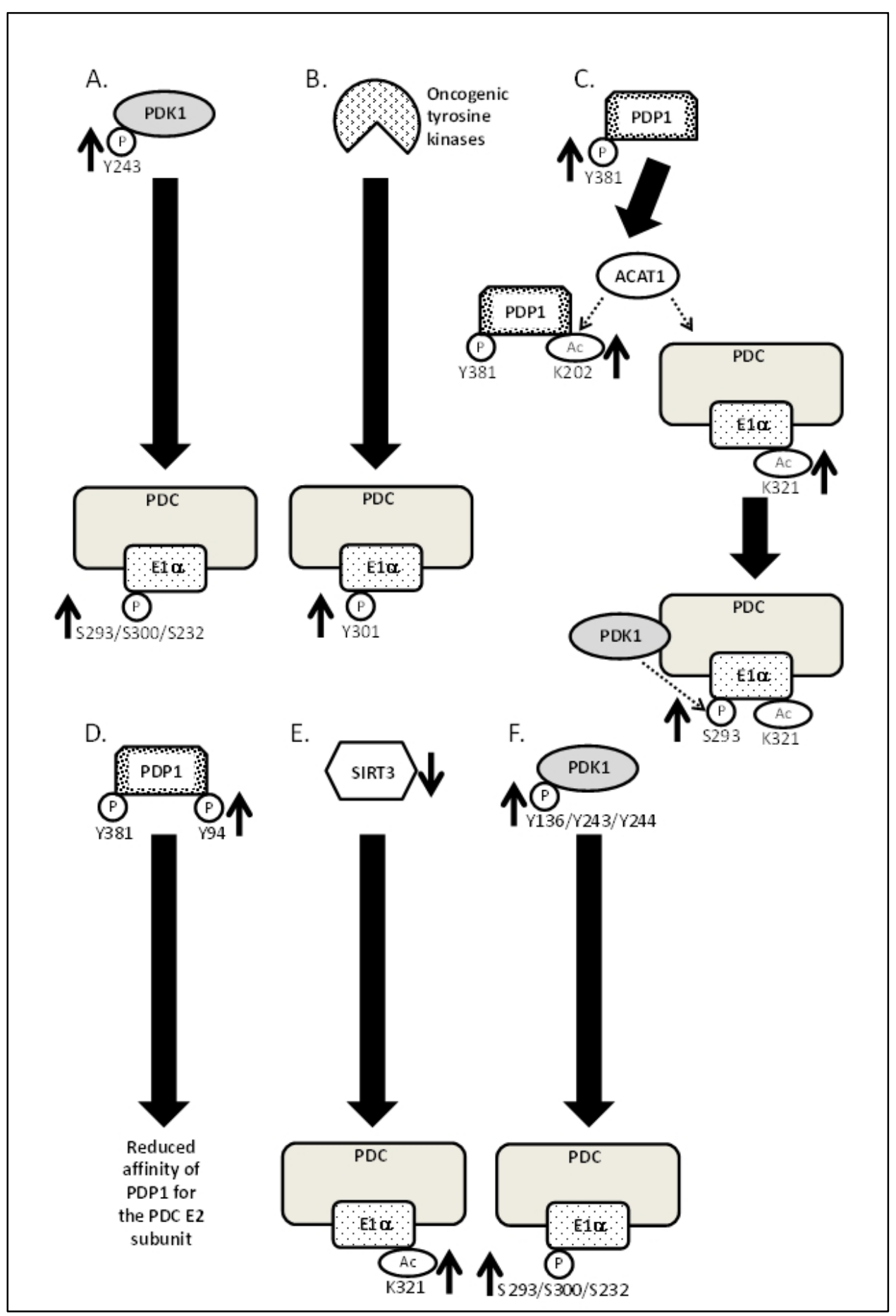

Figure 2: Mechanisms involved in decreasing PDC activity. (A) PDK1 is phosphorylated on Tyr243 leading to increased phosphorylation of the PDC E1 $\alpha$ subunit at Ser293, Ser300, or Ser232. (B) The PDC E1 $\alpha$ subunit can also be phosphorylated by multiple oncogenic tyrosine kinases on Tyr301 which blocks pyruvate substrate binding. (C) Tyr381 phosphorylation of PDP1 induces ACAT1 recruitment which subsequently acetylates PDP1 at Lys202, causing its deactivation, and the PDC E1 $\alpha$ subunit at Lys321 promoting PDC association with PDK1 and the subsequent phosphorylation of the former. (D) PDP1 phosphorylation at Try94 reduces its affinity for the PDC E2 subunit and, therefore, inhibits dephosphorylation of the latter. (E) Cancer cells may display decreased expression of SIRT3 which leads to increased acetylation of the PDC E1 $\alpha$ subunit at Lys321. (F) Oncogenic phosphorylation of PDK1 at Tyr136, Tyr243 or Tyr244 leads to increased phosphorylation of the PDC E1 $\alpha$ subunit at Ser293, Ser300, or Ser232. 


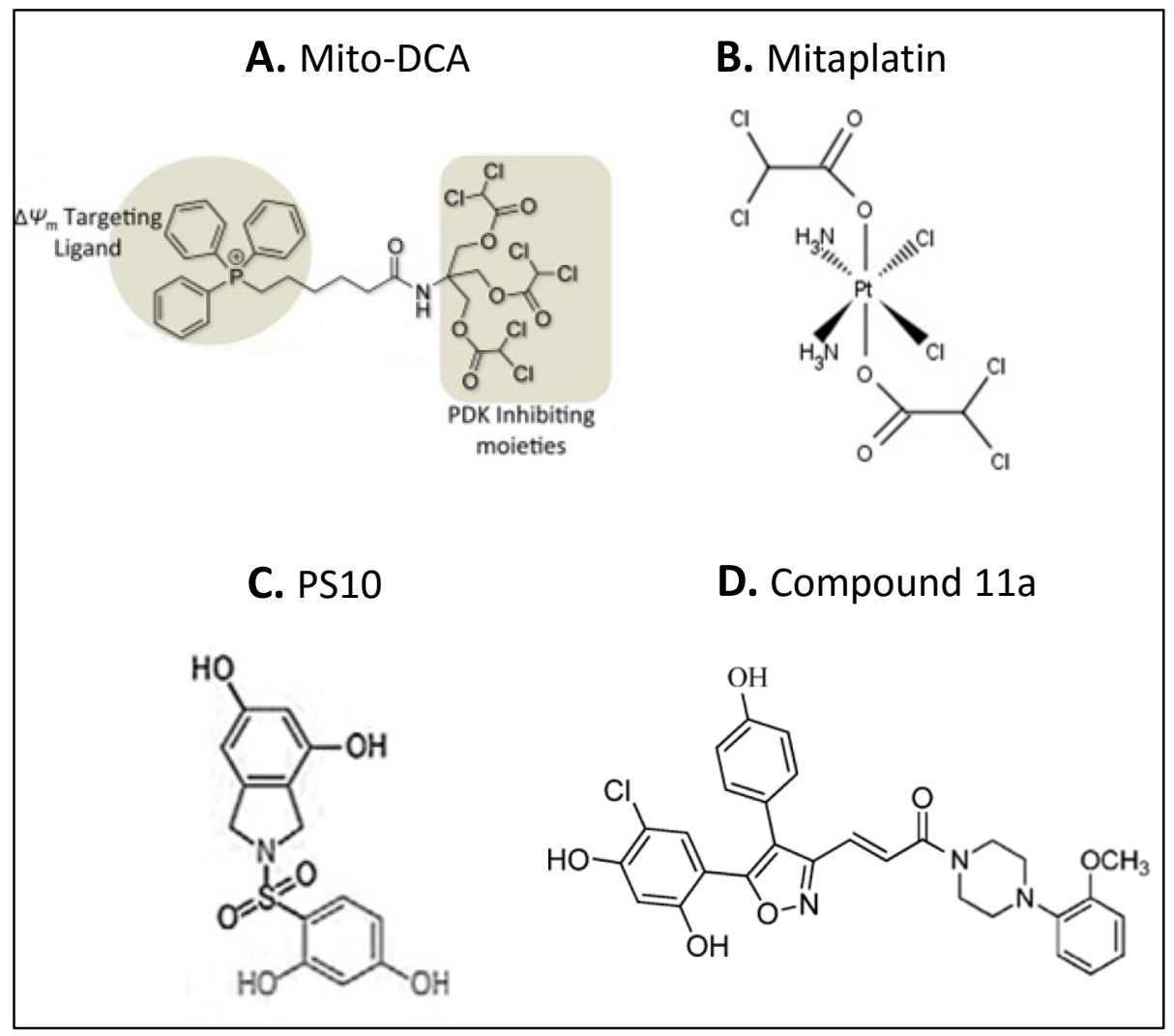

Figure 3: The structures of PDK inhibitors with potential for the treatment of cancer. (A) Mito-DCA is a DCA derivative which incorporates a lipophilic triphenylphosphonium cation linked to the DCA-derivative PDK inhibiting part of the molecule (both highlighted). (B) Mitaplatin is a fusion of a six-coordinate platinum (IV) complex synthesized from the widespread chemotherapeutic cisplatin, with two DCA moieties in the axial positions. (C) PS10 is based on the structure of the PDK ATP-binding pocket and is derived from a known Hsp90 inhibitor with a key substitution of the carbonyl group, linking the resorcinol and isoindoline moiety of the molecule, to a sulphonyl group. (D) Compound 11a is structured on a 4,5-diarylisoxazole-based Hsp90 inhibitor and is a 3-amide substituted isooxazole derivative.

\subsection{Pyruvate dehydrogenase kinases}

PDKs are serine-specific protein kinases which are intimately associated with the PDC through binding to the lipoyl domains of E2. These heterodimeric kinases are unrelated to cytoplasmic Ser/Thr/Tyr kinases and are composed of a catalytically active $\alpha$-subunit and a regulatory $\beta$-subunit; the $\alpha$-subunit is composed of eight sub domains, all of which are critical for the correct binding and orientation of ATP. ${ }^{10}$ Four mammalian isoforms of PDK differentially phosphorylate the specific serine residues of the PDC, conferring additional complexity to PDC regulation; PDK1 can phosphorylate all three sites, whilst PDK2, PDK3, and PDK4, can phosphorylate sites 1 and 2 only. ${ }^{\mathbf{1 1}}$

PDKs are subject to acute, allosteric stimulation by the reaction products of PDC (NADH and acetyl-CoA) by reduction of lipoyl moieties on the E2 lipoyl domains of $\mathrm{E}^{5}$. Reactant availability, namely pyruvate and ADP, promotes PDK inhibition and a combination of these effectors act synergistically. ${ }^{12}$ It follows that product/reactant ratios determine PDK activity by the proportion of active PDC (dephosphorylated) to inactive PDC (phosphorylated).
PDK1 may be regulated by oncogenic tyrosine phosphorylation such as that by oncogenic fibroblast growth factor receptor 1 . It has been demonstrated that PDK1 may be phosphorylated on at least three different tyrosine residues by tyrosine kinases (Tyr136, Tyr243, and Tyr244), which increases ATP binding affinity toward the active site and promotes association with PDC. ${ }^{13}$ Increased efficiency of PDK1 means that E1 $\alpha$ is inactivated by phosphorylation more efficiently by this oncogenic tyrosine kinase stimulation of PDK1.

\subsection{Pyruvate dehydrogenase phosphatases}

PDPs are heterodimeric enzymes composed of a catalytic and a regulatory subunit with two isoforms of the catalytic subunit identified in mammalian tissues; PDP1 and PDP2. Both PDP isoforms require $\mathrm{Mg}^{2+}$ for catalysis with PDP1 activity being more sensitive to cellular $\mathrm{Mg}^{2+}$ concentration than PDP2. PDP1 binds to the L2 domain of the PDC E2 subunit, facilitated by $\mathrm{Ca}^{2+}$ playing a 'bridging role'5 - thus, cellular $\mathrm{Ca}^{2+}$ concentration regulates PDP1 activity and, in turn, PDC activity. PDP2 binding to a lipoyl domain of E2 has not been detected and the isoform is not sensitive to $\mathrm{Ca}^{2+}$, 
though does require a higher concentration of $\mathrm{Mg}^{2+}$ than PDP1 for catalysis. Both PDP isoforms are capable of dephosphorylating each serine site and thus reactivating the PDC, though with diminutive variance in their activities for each site; in the order site $2>$ site $3>$ site $1 .^{14}$

Like PDKs, PDPs may be regulated by oncogenic tyrosine kinases such as ABL and JAK2. For example, Tyr94 phosphorylation on PDP1 reduces binding ability to lipoic acid of the E2 component of the PDC and therefore decreases association of PDP with PDC. ${ }^{15}$

\subsection{Acetylation and succinylation}

Acetylation of lysine residues constitutes a novel post-transcriptional modification which may rival phosphorylation as a major regulator of many, if not all, metabolic pathways. ${ }^{16} \mathrm{~A}$ number of lysine acetylation sites have been identified on PDC E1 $\alpha$ in cancer cells, which complement the phosphorylation-mediated regulation of the PDC. ${ }^{17}$ The acetylation status of PDC E1 $\alpha$ influences activity of the complex due to the interactions of lysine acetylation sites with PDKs and PDPs. The mitochondrial acetyltransferase ACAT1 and the mitochondrial deacetylase sirtuin 3 (SIRT3) have been implicated in this respect.18 In normal, differentiated cells, PDC is active with E1 $\alpha$ being associated with PDP1 and SIRT3. However, cancer cells demonstrate inactive PDC with E1 $\alpha$ associated with PDK1. In this oncogenic process, Y381 phosphorylation causes the dissociation of SIRT3 and the recruitment of ACAT1. Acetylation of PDP1 and E1 $\alpha$ by ACAT1 causes the dissociation of PDP1 and the recruitment of active PDK1, with a subsequent phosphorylation event by PDK1 to render the PDC inactive. Furthermore, many human cancers are associated with a SIRT3 deficiency, and mice deficient in SIRT3 have been shown to spontaneously develop breast cancer. ${ }^{18}$

Reversible succinylation also constitutes a post-transcriptional modification of metabolic enzymes and a number of lysine succinylation sites have been identified on the E1 $\alpha, E 1 \beta, E 2$, and E3 subunits of PDC. ${ }^{19}$, ${ }^{20}$ Most of the succinylation sites do not overlap with the aforementioned acetylation sites, suggesting divergent regulatory mechanisms. Indeed, SIRT5 has been shown to negatively regulate PDC by means of succinylation, suppressing the enzyme's metabolic role. ${ }^{19}$

\subsection{Transcriptional regulation}

In addition to the post-transcriptional regulation of PDC, long-term regulation of the enzyme complex may be achieved via transcriptional control. Indeed, in a number of disease, endocrine and nutritional states, the activity of PDC has been shown to be regulated by the ratio of PDKs and PDPs determined at the transcriptional level.21 In the context of cancer, PDK1 expression has been shown to be upregulated by the oncogenes hypoxia-inducible factor- $1 \alpha$ (HIF- $1 \alpha$ and c-Myc which inactivate PDC by phosphorylation to essentially achieve global inhibition of the complex and, therefore, define an oxidative metabolic profile. ${ }^{22}$

\section{The Warburg effect and cancer metabolism}

The heterogeneity and adaptability of cancer cells presents immense challenges in cancer treatment and chemotherapeutic targeting of cancer cells has traditionally been non-specific, affecting cellular processes or components essential to normal cells. The identification of pathways, phenotypes, or genotypes unique to cancer cells may drastically improve prospects for efficacious, selective treatments. However, molecular characteristics of even histologically identical cancers prove highly dissimilar, even within the same tumour. ${ }^{23}$

Under normal, aerobic, physiological conditions, cells meet their energy requirements by the production of ATP in the mitochondria via oxidative phosphorylation. Alternatively, under hypoxic conditions, normal cells derive their ATP from the conversion of glucose to lactate. The Pasteur effect describes the cell-autonomous adaptation of most mammalian cells to the presence of oxygen, characterized by a decreased rate of glycolysis and an increased rate of oxidative phosphorylation. ${ }^{24}$ In direct contradiction, Warburg observed that cancer cells shift to aerobic glycolysis as the main source of ATP, with an associated increased uptake of glucose and enhanced lactate production, even under normoxia. ${ }^{1}$ These seminal findings were confirmed by a number of different researchers which extended Warburg's in vitro model into in vivo models, though a number of studies may be questionable. ${ }^{25,26}$ For example, in a study investigating glucose utilization of a Novikoff hepatoma, it was concluded that lactate production was four times higher than in normal liver cells. However, the glycolytic contribution of ATP could not be deduced in this study as oxygen consumption was not measured and, therefore, did not constitute a valid reflection of total oxidative ATP production. ${ }^{27}$

Warburg later proposed that tumour cells possess an intrinsic defect of oxidative metabolism, explaining the observed increased glycolytic flux as a compensatory mechanism. ${ }^{28}$ Whilst research has repeatedly, though often questionably, shown increased aerobic glycolysis in many tumour cell types, a metabolic defect of cancer cells has not been established. Rather, research supports the existence of both a functional TCA cycle and pronounced aerobic glycolysis, demonstrating a priority of metabolic activity for biosyntheses as opposed to complete glucose oxidation. ${ }^{29}$ Although the phenomenon is not universal to all cancer types ${ }^{30}$, it is sufficiently well established that it often forms the basis for cancer diagnostics using the glucose analogue tracer ${ }^{\mathbf{1 8}}$ fluorodeoxyglucose in tandem with positron emission tomography (FDG-PET) ${ }^{31}$, though there are a number of 
established factors which may confound results such as glucose concentration, tissue density, and fibrosis ${ }^{32}$. However, the assumption that FDG-PET functions on the basis of increased aerobic glycolysis specifically appears to be unreasoned.

Because aerobic glycolysis is much less efficient than oxidative phosphorylation at generating ATP, cancer cells upregulate glucose receptor and glycolytic enzyme gene transcription in a bid to compensate for this inefficiency and meet their increasing ATP demands. This metabolic profile also favours the reduced funneling of metabolic intermediates to oxidative catabolism within the mitochondria and, instead, toward anabolic processes. ${ }^{33}$ The accumulation of both glycolytic and TCA cycle intermediates has been shown to enhance cancer malignancy by the promotion of angiogenesis, adaptability to hypoxic conditions, metastasis, and immune-evasion. ${ }^{34}$ Thus, the Warburg effect provides benefits to both cell bioenergetics and biosyntheses. Accordingly, increased glucose uptake and high lactate levels are correlated with poor cancer patient prognosis and increased tumour aggressiveness ${ }^{35,36}$, though it is important to consider that the Warburg phenotype is not unique to cancer cells, but is also observed in non-cancer cells during rapid proliferation. ${ }^{37}$

The Warburg metabolic phenotype occurs early in tumourigenesis. It has been proposed that tumour cells must rely on anaerobic glycolysis as a primary ATP source in the hypoxic microenvironment during early tumourigenesis ${ }^{38}$; the transient hypoxia of a developing tumour mass cannot meet oxygen demands to carry out oxidative metabolism due to local vasculature restrictions. Tumour cells may evade hypoxia-mediated cell death by the mutation or decreased expression of p53. ${ }^{39}$ The up regulation of glycolysis is achieved via the hypoxic induction of HIF-1 which stimulates key steps in glycolysis whilst also regulating genes controlling angiogenesis, cell survival, and cell invasion. HIF-1 is a heterodimer of a constitutively expressed HIF-1 $\beta$ subunit and a regulated HIF- $1 \alpha$ subunit. Under normoxic conditions, HIF-1 is rapidly degraded via the ubiquitin-proteasomal pathway; HIF prolyl hydroxylases hydroxylate Pro402 and Pro564 of HIF-1 $\alpha$ promoting association of HIF-1 with the tumour suppressor protein Von Hippel-Lindau (pVHL) which acts as a ubiquitin ligase substrate recognition component of an E3 ubiquitin ligase. ${ }^{40}$ The response to hypoxia is, in part, mobilized by the activation of HIF-1 by preventing its degradation; this mediates a switch from oxidative to glycolytic metabolism by regulating expression of many glycolytic enzymes, glucose transporters, and mitochondrial enzymes. ${ }^{41}$ HIF-1 also activates the transcription of genes controlling angiogenesis, erythropoiesis, cell survival, and cell migration ${ }^{42}$ which can amplify malignant progression. However, the stabilization of HIF-1 may also be observed under normoxic conditions suggesting that other factors, such as hormones, growth factors, or oncogenes, may cause HIF-1 stabilization and promotion of the glycolytic phenotype. ${ }^{43}$ One of the most common molecular abnormalities, loss of p53, directly induces pyruvate dehydrogenase kinase expression and enhances HIF-1 $\alpha$ transcription and stability. ${ }^{44,45}$ Alternatively, mutations in tumour suppressor VHL may result in the constitutive activation of HIF-1 $\alpha$. The collective action of many oncogenes may emit "pseudo-hypoxic" signals establishing a feed-forward feedback loop to sustain the glycolytic phenotype.

In addition to the up regulation of glucose, HIF-1 has been shown to enhance expression of PDK1.46 By diverting pyruvate towards glycolysis, the tumour cell mitochondria are essentially deprived of their "fuel" which, therefore, suppresses mitochondrial activity, concomitantly enhancing apoptotic resistance. Mitochondria-mediated apoptosis depends on the efflux of pro-apoptotic mediators, such as cytochrome $c$, from the mitochondria through the voltage- and redox-gated mitochondrial permeability transition pore (MPTP). The MPTP is a megachannel which includes the voltage-dependent anion channel (VDAC), requiring significant mitochondrial depolarization for opening. ${ }^{47}$ Suppressed mitochondrial function promotes hyperpolarization of the mitochondrial membrane and a decrease in the generation of reactive oxygen species ${ }^{48}$; both of which contribute to an increase in the opening threshold required for the MPTP, which suppresses apoptosis and, again, promotes tumour growth. The suppression of mitochondria can also stabilize HIF- $1 \alpha$. This is because the TCA cycle product $\alpha$-ketoglutarate, a required co-factor for HIF- $1 \alpha$ destabilization by prolyl hydroxylases, is decreased. ${ }^{49}$

Whilst the notion of HIF-1 $\alpha$ stability has much supporting evidence, some cancers demonstrate glycolytic metabolism before exposure to hypoxia. Leukaemic cells, for example, are subject to higher oxygen concentrations than cells of other tissues yet are highly glycolytic. ${ }^{50}$

In summary, the fate of glucose in metabolism is largely dependent on the gate-keeper pyruvate dehydrogenase complex at the interface of glycolysis and the TCA cycle. To stress the importance of PDC activity, replacing endogenous PDK1 with a mutated form in which the phosphorylated tyrosine residues are mutated, causes a shift in the metabolic profile of cancer cells toward oxidative metabolism; suggesting a reversion of the Warburg effect.13 This warrants PDC, and particularly PDK, of great interest in the potential therapeutic reversion of the cancer cell Warburg effect as a means of cancer therapy. 


\section{Therapeutic targeting of pyruvate dehydrogenase kinase in cancer}

\subsection{Dichloroacetate}

Dichloroacetate (DCA) is a small, 150 Da, well-established inhibitor of PDK which can penetrate most tissues following oral administration, including the central nervous system, entering cells via the monocarboxylate and pyruvate carrier systems and concentrating in the mitochondria. ${ }^{51}$ In a dose-dependent fashion, DCA inhibits PDK at concentrations of $10-250 \mu \mathrm{M}$ subsequently activating PDH. ${ }^{52} \mathrm{PDK}$ isoforms have a mostly conserved pocket to which DCA binds; PDK2 is the most ubiquitously expressed isoform and is most sensitive to inhibition by DCA. ${ }^{53}$ In PDK2, DCA occupies the same binding site as pyruvate; the carboxylate group of DCA forms a salt-bridge with Arg154 in the N-terminal domain of the enzyme, with the chlorine atoms residing in a hydrophobic region of the pocket. ${ }^{54}$ Suppression of PDK2 expression using siRNA mimics the action of DCA, thereby confirming inhibition of the enzyme as the major pharmacodynamic mechanism of DCA. ${ }^{55}$

Historically, DCA was used for ameliorating the symptoms of lactic acidosis associated with a number of congenital mitochondrial diseases in children. ${ }^{56}$ Over the past few decades, chronic DCA administration has been clinically tested in adults and children in a number of disease states associated with lactic acidosis including diabetes mellitus and pulmonary arterial hypertension. ${ }^{57-59}$ More recently, the clear anti-neoplastic activity of DCA has been extensively studied in a number of investigations involving different tissues including those from prostate cancer ${ }^{\mathbf{6 0}}$, endometrial cancer ${ }^{61}$ and breast cancer ${ }^{62}$. In fact, in vivo studies have demonstrated the reversion of an aggressive metastatic breast cancer corroborate with the anti-neoplastic activity of DCA. ${ }^{62}$ However, the published research sometimes reports contradictory in vitro and in vivo effects. ${ }^{63}$

The activation of PDC by means of DCA inhibition of PDK causes an increased carbon flux through PDC and an increased mitochondrial production of NADH. The electron-donor NADH is a substrate of electron-transport chain (ETC) complex-I, and thus leads to an increased production of reactive oxygen species (ROS).64 Furthermore, it has been proposed that increased ROS production may lead to oxidative damage in the ETC causing restricted mitochondrial proton efflux, in turn causing a decrease in membrane potential.55 As discussed earlier in this article, hyperpolarization of the mitochondrial membrane is partly responsible for the suppression of mitochondrial function in cancer cells. Through the sustained complex-I generation of ROS, significantly decreased mitochondrial membrane potential allows the opening of the voltage-sensitive MPTP, allowing efflux of pro-apoptotic proteins such as cytochrome $c$ and initiation of apoptosis via caspase activation. Additionally, through the generation of ROS, DCA essentially reverses the "pseudo-hypoxic" phenotype of cells in vivo by inhibition of $\mathrm{PDK}^{65}$ and suppresses normoxic activation of HIF-1 $\alpha$. Increased ROS also increase the activity of redox-sensitive tumour suppressors such as p53 which, in tandem with $\alpha$-ketoglutarate, lead to decreased stabilization of HIF-1 $\alpha^{66}$. Whilst the ROS-mediated induction of apoptosis is generally accepted as the leading mechanism of DCA action, cell cycle arrest and the induction of autophagy have been demonstrated in human colorectal and prostate carcinoma cell lines, and HT29 tumour xenografts following DCA treatment. ${ }^{67}$ It should, however, be noted that the in vitro results in some of these studies are somewhat questionable from a physiological point of view due to the high concentrations of DCA used (75-100 mM).

In a clinical trial of five patients with glioblastoma multiforme, an eclectic combination of DCA, temozolomide, radiation, and surgery were used in the treatment regime. ${ }^{68}$ With an emphasis on ex vivo analyses of tumour cells, the authors demonstrated changes to the mitochondrial membrane potential, increased levels of mitochondrial ROS, and increased apoptosis. Further investigations revealed suppressed HIF-1 $\alpha$ expression, enhanced p53 activation and decreased angiogenesis both in vitro and in vivo demonstrating the multitude of downstream effects of PDK inhibition. One patient with recurrent disease died from complications of brain oedema three months after starting DCA therapy while the remaining four of the five patients showed promising results.

Many of the concerns raised over potential patient toxicity of DCA are based on data obtained from in vitro or in vivo studies in which DCA doses administered were many orders of magnitude higher than those to which humans are exposed in treatments. Our patient tolerability knowledge of DCA dosing generally comes from publications investigating the drug as a treatment for individuals with mitochondrial-linked lactic acidosis. Specifically, DCA has been used to treat older adolescents and adults (mean age at study entry; 30 years) with the common A3243G mutation in mitochondrial DNA that gives rise to the syndrome of mitochondrial encephalomyopathy, lactic acidosis and stroke-like episodes (MELAS). ${ }^{69}$ Unfortunately, using a twice daily dose of $12.5 \mathrm{mg} / \mathrm{kg}$, the study had to be terminated prematurely because of an undue incidence of worsening or new onset peripheral neuropathy. A later study examined the same dosing regime in younger children (mean age at study entry; 5.6 years) with congenital lactic acidosis (CLA). ${ }^{51}$ In contrast to the earlier study in older patients, the authors concluded that there was good tolerability of DCA and no significant differences in the severity or frequency of 
adverse events between the drug and placebo groups over a six month treatment period. However, the same group subsequently showed that, after long term drug treatment (10 years) some significant worsening in peripheral neuropathy was observed in the DCA-treated cohort. $^{70}$ Interestingly, the rate of adverse effect onset seems directly related to patient age with adults and young adolescents being more rapidly affected than children. Accordingly, DCA plasma clearance has been shown to be inversely associated with age in humans. ${ }^{71}$ In summary, DCA does seem to exhibit some detrimental effects in humans in terms of peripheral neuropathy. However, given that the above studies concluded that these effects were largely reversible following cessation of drug treatment and the fact that many current chemotherapeutic drugs cause similar effects, perhaps this is a relatively small price to pay for the use of DCA in cancer treatment.

The clinical history of DCA warrants its further investigation as a cancer monotherapy by targeting a fundamental and unique property of many cancer cells, giving an apparent, selective reversal of the Warburg metabolic phenotype. However, given the metabolic and apoptotic effects of DCA, it could be given concurrently with other chemotherapeutics to potentially increase their effectiveness or reduce required dosages, thereby limiting toxicity. ${ }^{72}$ Accordingly, co-treatment with DCA, tamoxifen and omeprazole displayed a synergistic enhancement of anti-tumour activity. ${ }^{73}$ Whilst chemotherapy resistance is a notorious characteristic of many cancers, 5-fluorouracil (5-FU) resistant hypoxic gastric cancer cells were successfully re-sensitized by co-treatment with DCA and 5-FU.74 Similarly, the combination of the same two drugs has been shown to exert a synergistic anti-tumour effect in colorectal cancer. ${ }^{75}$ The success of DCA as a monotherapy and in tandem with many alternative chemotherapeutics has been exploited by chemical modifications and direct conjugation of DCA with several chemotherapeutics.

\subsection{Dichloroacetate derivatives and other pyruvate dehydrogenase kinase inhibitors}

Mito-DCA (Figure 3A) is a DCA derivative which incorporates a lipophilic triphenylphosphonium cation through a biodegradable linker. ${ }^{76}$ This incorporation purports to enhance the potency and cancer cell specificity of DCA by three orders of magnitude. Whilst appearing to have no significant metabolic effects on normal cells, Mito-DCA did cause a reversion of the Warburg effect and induction of apoptosis in cancer cells with dysfunctional mitochondria.

Mitaplatin (Figure 3B) is a fusion of a six-coordinate platinum (IV) complex synthesized from the existing chemotherapeutic cisplatin, with two DCA moieties in the axial positions. ${ }^{77}$ Once within the cell, mitaplatin dissociates into its respective molecules due to the negative intracellular redox potential; cisplatin targets the nucleus where it forms intra-strand nucleotide crosslinks, whilst DCA targets the mitochondria reversing the Warburg effect and promoting apoptosis. In a number of different cancer cell lines, mitaplatin has proven to exceed the efficacy of cisplatin alone. ${ }^{77}$ Interestingly, mitaplatin has also proven to be more efficacious than in studies where DCA and cisplatin were co-administered but as separate entities. ${ }^{78}$ This may permit the administration of a lower effective dose of Mitaplatin than when either of the separate compounds are employed thereby alleviating some of the peripheral neurotoxicity side effects associated with these drugs.

PS10 (Figure 3C) is highly specific PDK inhibitor based on the structure of the PDK ATP-binding pocket conserved amongst the GHKL ATPase/kinase superfamily which also includes heat-shock protein 90 (Hsp90). PS10 was derived from a known Hsp90 inhibitor to form a highly specific PDK inhibitor with a key substitution of the carbonyl group, linking the resorcinol ring and isoindoline moieties of the molecule, to a sulphonyl group. ${ }^{79}$ In vivo studies with PS10 demonstrated significantly greater PDC activity levels, supporting inhibition of PDK as the mode of action of the molecule and its potential usage in reversal of the Warburg effect. Another structure-guided development of a highly specific PDK inhibitor, based on the conserved ATP-binding pocket, is compound 11a (Figure 3D) which is structured on a 4,5-diarylisoxazole-based Hsp90 inhibitor and is a 3-amide substituted isoxazole derivative. ${ }^{80}$ The dual inhibition of Hsp90 and PDC may provide some advantage as a cancer therapeutic, though it is important to consider similarities of nucleotide-binding pockets in other kinases which may result in unwanted cytotoxic effects.

\section{Conclusions and future directions}

Warburg's work did not translate into cancer therapies for many decades as it was long believed that the unique metabolic phenotype of cancer cells was a consequence of, but not a major contributor to or cause of, cancer. However, metabolic oncology is now a thriving field in the battle against cancer.

The number of cancer types and the experiments employed to date are too limited to make generalized conclusions on the efficacy of DCA in cancer therapy. DCA has a tremendous clinical profile in the therapeutic metabolic modulation of mitochondrial diseases and has proven a promising cancer therapy in a number of studies from the past decade. This warrants thorough exploration into DCA and its derivatives as means of attaining higher efficacy and reduced toxicity in combination therapies with current, standard cancer treatments which are often non-selective for cancerous cells. Perhaps the fact that investigations into DCA are stagnating is due to the intrinsic generic nature of DCA, meaning it cannot be patented. This creates financial 
barriers to truly exploiting the clinical efficacy of the drug in cancer therapy.

\section{Conflict of interest}

The authors declare that they have no conflicts of interest. The authors alone are responsible for the content and writing of the paper.

\section{Abbreviations}

ACAT1 $=$ acetyl-CoA acetyltransferase 1;

ATP = adenosine 5' -triphosphate;

CoA = Coenzyme A;

DCA = dichloroacetate;

ETC = electron-transport chain;

5-FU = 5-fluorouracil;

$\mathrm{FAD} / \mathrm{FADH}_{2}=$ flavin adenine dinucleotide / reduced;

HIF-1 = hypoxia inducible factor-1;

Hsp90 = heat-shock protein 90;

JAK2 = janus kinase 2;

MPTP = mitochondrial permeability transition pore;

NAD / NADH = nicotinamide adenine dinucleotide / reduced;

PDC = pyruvate dehydrogenase complex;

PDK = pyruvate dehydrogenase kinase;

PDP = pyruvate dehydrogenase phosphatase;

pVHL = protein Von Hippel-Lindau;

ROS = reactive oxygen species;

SIRT3 = NAD-dependent deacetylase sirtuin 3;

SIRT5 = NAD-dependent deacetylase sirtuin 5;

TCA = tricarboxylic acid;

TPP = thiamine pyrophosphate;

VDAC $=$ voltage-dependent anion channel

\section{References}

1. Warburg O, Posener K, Negelein E. Ueber den stoffwechsel der tumoren. Biochemische Zeitschrift. 1923;152: 319.

2. Berg J, Tymoczko J, Stryer L. Biochemistry. $7^{\text {th }}$ revised international edition ed.: Palgrave Macmillan; 2011.

3. Ciszak EM, Korotchkina LG, Dominiak PM, et al. Structural basis for flip-flop action of thiamin pyrophosphate-dependent enzymes revealed by human pyruvate dehydrogenase. J Biol Chem. 2003;278(23): 21240-6.

4. Zhou ZH, McCarthy DB, O'Connor CM, et al. The remarkable structural and functional organization of the eukaryotic pyruvate dehydrogenase complexes. Proc Natl Acad Sci U S A. 2001;98(26):14802-7.

5. Roche TE, Baker JC, Yan X, et al. Distinct regulatory properties of pyruvate dehydrogenase kinase and phosphatase isoforms. Prog Nucleic Acid Res Mol Biol. 2001.70:33-75.
6. Korotchkina LG, Patel MS. Mutagenesis studies of the phosphorylation sites of recombinant human pyruvate dehydrogenase. Site-specific regulation. J Biol Chem. 1995; 270(24):14297-304.

7. Korotchkina LG, Patel MS. Probing the mechanism of inactivation of human pyruvate dehydrogenase by phosphorylation of three sites. J Biol Chem. 2001;276(8):5731-8.

8. Seifert F, Ciszak E, Korotchkina L, et al. Phosphorylation of serine 264 impedes active site accessibility in the E1 component of the human pyruvate dehydrogenase multienzyme complex. Biochemistry. 2007;46(21):6277-87.

9. Fan J, Kang HB, Shan C, et al. Tyr-301 phosphorylation inhibits pyruvate dehydrogenase by blocking substrate binding and promotes the Warburg effect. J Biol Chem. 2014;289(38):26533-41.

10. Steussy CN, Popov KM, Bowker-Kinley MM, et al. Structure of pyruvate dehydrogenase kinase. Novel folding pattern for a serine protein kinase. J Biol Chem. 2001;276(40): 37443-50.

11. Korotchkina LG, Patel MS. Site specificity of four pyruvate dehydrogenase kinase isoenzymes toward the three phosphorylation sites of human pyruvate dehydrogenase. J Biol Chem. 2001;276(40):37223-9.

12. Pratt ML, Roche TE. Mechanism of pyruvate inhibition of kidney pyruvate dehydrogenasea kinase and synergistic inhibition by pyruvate and ADP. J Biol Chem. 1979;254(15):7191-6.

13. Hitosugi T, Fan J, Chung TW, et al. Tyrosine phosphorylation of mitochondrial pyruvate dehydrogenase kinase 1 is important for cancer metabolism. Mol Cell. 2011;44(6): 864-77.

14. Karpova T, Danchuk S, Kolobova E, et al. Characterization of the isozymes of pyruvate dehydrogenase phosphatase: implications for the regulation of pyruvate dehydrogenase activity. Biochim Biophys Acta. 2003; 1652(2): 126-35.

15. Shan C, Kang HB, Elf S, et al. Tyr-94 phosphorylation inhibits pyruvate dehydrogenase phosphatase 1 and promotes tumor growth. J Biol Chem. 2014; 289(31): 21413-22.

16. Smith BC, Settles B, Hallows WC, et al. SIRT3 substrate specificity determined by peptide arrays and machine learning. ACS Chem Biol. 2011; 6(2): 146-57.

17. Fan J, Shan C, Kang HB, et al. Tyr phosphorylation of PDP1 toggles recruitment between ACAT1 and SIRT3 to regulate the pyruvate dehydrogenase complex. Mol Cell. 2014; 53(4): 534-48.

18. Kim HS, Patel K, Muldoon-Jacobs K, et al. SIRT3 is a mitochondria-localized tumor suppressor required for maintenance of mitochondrial 
integrity and metabolism during stress. Cancer Cell. 2010; 17(1): 41-52.

19. Park J, Chen Y, Tishkoff DX, et al. SIRT5-mediated lysine desuccinylation impacts diverse metabolic pathways. Mol Cell. 2013; 50(6): 919-30.

20. Zhang Z, Tan M, Xie Z, et al. Identification of lysine succinylation as a new post-translational modification. Nat Chem Biol. 2011; 7(1): 58-63.

21. Sugden MC, Holness MJ. Recent advances in mechanisms regulating glucose oxidation at the level of the pyruvate dehydrogenase complex by PDKs. Am J Physiol Endocrinol Metab. 2003; 284(5): E855-62.

22. Kim JW, Gao P, Liu YC, et al. Hypoxia-inducible factor 1 and dysregulated c-Myc cooperatively induce vascular endothelial growth factor and metabolic switches hexokinase 2 and pyruvate dehydrogenase kinase 1. Mol Cell Biol. 2007; 27(21): 7381-93.

23. Marusyk A, Almendro V, Polyak K. Intra-tumour heterogeneity: a looking glass for cancer? Nat Rev Cancer. 2012; 12(5): 323-34.

24. Racker E. History of the Pasteur effect and its pathobiology. Mol Cell Biochem. 1974; 5(1-2): 17-23.

25. Cori C, Cori G. The carbohydrate metabolism of tumours. I. The free sugar, lactic acid, and glycogen content of malignant tumours. J Biol Chem. 1925; 65: 397-405.

26. Cori C, Cori G. The carbohydrate metabolism of tumours. II. Changes in the sugar, lactic acid, and co-combing power of blood passing through a tumour. J Biol Chem. 1925; 65: 397-405.

27. Ashmore J, Weber G, Landau BR. Isotope studies on the pathways of glucose-6-phosphate metabolism in the Novikoff hepatoma. Cancer Res. 1958; $18(8$ Part 1): 974-9.

28. Warburg 0 . On respiratory impairment in cancer cells. Science. 1956; 124(3215): 269-70.

29. DeBerardinis RJ, Mancuso A, Daikhin E, et al. Beyond aerobic glycolysis: transformed cells can engage in glutamine metabolism that exceeds the requirement for protein and nucleotide synthesis. Proc Natl Acad Sci U S A. 2007; 104(49): 19345-50.

30. Funes JM, Quintero M, Henderson S, et al. Transformation of human mesenchymal stem cells increases their dependency on oxidative phosphorylation for energy production. Proc Natl Acad Sci U S A. 2007; 104(15): 6223-8.

31. Hawkins RA, Phelps ME. PET in clinical oncology. Cancer Metastasis Rev. 1988; 7(2): 119-42.

32. Pauwels EK, Sturm EJ, Bombardieri E, et al. Positron-emission tomography with [18F]fluorodeoxyglucose. Part I. Biochemical uptake mechanism and its implication for clinical studies. J Cancer Res Clin Oncol. 2000; 126(10): 549-59.

33. DeBerardinis RJ, Lum JJ, Hatzivassiliou G, et al. The biology of cancer: metabolic reprogramming fuels cell growth and proliferation. Cell Metab. 2008; 7(1): 11-20.

34. Draoui N, Feron O. Lactate shuttles at a glance: from physiological paradigms to anti-cancer treatments. Dis Model Mech. 2011; 4(6): 727-32.

35. Kunkel M, Reichert TE, Benz P, et al. Overexpression of Glut- 1 and increased glucose metabolism in tumors are associated with a poor prognosis in patients with oral squamous cell carcinoma. Cancer. 2003; 97(4): 1015-24.

36. Walenta S, Schroeder T, Mueller-Klieser W. Lactate in solid malignant tumors: potential basis of a metabolic classification in clinical oncology. Curr Med Chem. 2004; 11(16): 2195-204.

37. Roos D, Loos JA. Changes in the carbohydrate metabolism of mitogenically stimulated human peripheral lymphocytes. II. Relative importance of glycolysis and oxidative phosphorylation on phytohaemagglutinin stimulation. Exp Cell Res. 1973; 77(1): 127-35.

38. Gatenby RA, Gillies RJ. Why do cancers have high aerobic glycolysis? Nat Rev Cancer. 2004; 4(11): 891-9.

39. Moll UM, Schramm LM. p53--an acrobat in tumorigenesis. Crit Rev Oral Biol Med. 1998; 9(1): 23-37.

40. Hagg M, Wennstrom S. Activation of hypoxia-induced transcription in normoxia. Exp Cell Res. 2005; 306(1): 180-91.

41. Semenza GL. Hypoxia-inducible factors in physiology and medicine. Cell. 2012; 148(3): 399-408.

42. Harris AL. Hypoxia--a key regulatory factor in tumour growth. Nat Rev Cancer. 2002; 2(1): 38-47.

43. Richard DE, Berra E, Pouyssegur J. Nonhypoxic pathway mediates the induction of hypoxia-inducible factor 1 alpha in vascular smooth muscle cells. J Biol Chem. 2000; 275(35): 26765-71.

44. Contractor T, Harris CR. p53 negatively regulates transcription of the pyruvate dehydrogenase kinase Pdk2. Cancer Res. 2012; 72(2): 560-7.

45. Ravi R, Mookerjee B, Bhujwalla ZM, et al. Regulation of tumor angiogenesis by p53-induced degradation of hypoxia-inducible factor 1alpha. Genes Dev. 2000; 14(1): 34-44.

46. Kim JW, Tchernyshyov I, Semenza GL, et al. HIF-1-mediated expression of pyruvate dehydrogenase kinase: a metabolic switch 
required for cellular adaptation to hypoxia. Cell Metab. 2006; 3(3): 177-85.

47. Zamzami N, Kroemer G. The mitochondrion in apoptosis: how Pandora's box opens. Nat Rev Mol Cell Biol. 2001; 2(1): 67-71.

48. Hernandez-Garcia D, Wood CD, Castro-Obregon $\mathrm{S}$, et al. Reactive oxygen species: A radical role in development? Free Radic Biol Med. 2010; 49(2): 130-43.

49. Loenarz C, Schofield CJ. Expanding chemical biology of 2-oxoglutarate oxygenases. Nat Chem Biol. 2008; 4(3): 152-6.

50. Gottschalk S, Anderson N, Hainz C, et al. Imatinib (STI571)-mediated changes in glucose metabolism in human leukemia BCR-ABL-positive cells. Clin Cancer Res. 2004; 10(19): 6661-8.

51. Stacpoole PW, Kerr DS, Barnes C, et al. Controlled clinical trial of dichloroacetate for treatment of congenital lactic acidosis in children. Pediatrics. 2006; 117(5): 1519-31.

52. Stacpoole PW. The pharmacology of dichloroacetate. Metabolism. 1989; 38(11): 1124-44.

53. Bowker-Kinley MM, Davis WI, Wu P, et al. Evidence for existence of tissue-specific regulation of the mammalian pyruvate dehydrogenase complex. Biochem J. 1998; 329 ( Pt 1): 191-6.

54. Knoechel TR, Tucker AD, Robinson CM, et al. Regulatory roles of the $\mathrm{N}$-terminal domain based on crystal structures of human pyruvate dehydrogenase kinase 2 containing physiological and synthetic ligands. Biochemistry. 2006; 45(2): 402-15.

55. Bonnet S, Archer SL, Allalunis-Turner J, et al. A mitochondria-K+ channel axis is suppressed in cancer and its normalization promotes apoptosis and inhibits cancer growth. Cancer Cell. 2007; 11(1): 37-51.

56. Stacpoole PW, Henderson GN, Yan Z, et al. Clinical pharmacology and toxicology of dichloroacetate. Environ Health Perspect. 1998; 106 Suppl 4: 989-94.

57. McMurtry MS, Bonnet S, Wu X, et al. Dichloroacetate prevents and reverses pulmonary hypertension by inducing pulmonary artery smooth muscle cell apoptosis. Circ Res. 2004; 95(8): 830-40.

58. Stacpoole PW, Lorenz AC, Thomas RG, et al. Dichloroacetate in the treatment of lactic acidosis. Ann Intern Med. 1988; 108(1): 58-63.

59. Stacpoole PW, Moore GW, Kornhauser DM. Metabolic effects of dichloroacetate in patients with diabetes mellitus and hyperlipoproteinemia. N Engl J Med. 1978; 298(10): 526-30.

60. Cao W, Yacoub S, Shiverick KT, et al. Dichloroacetate (DCA) sensitizes both wild-type and over expressing Bcl-2 prostate cancer cells in vitro to radiation. Prostate. 2008; 68(11): 1223-31.

61. Wong JY, Huggins GS, Debidda M, et al. Dichloroacetate induces apoptosis in endometrial cancer cells. Gynecol Oncol. 2008; 109(3): 394-402.

62. Sun RC, Fadia M, Dahlstrom JE, et al. Reversal of the glycolytic phenotype by dichloroacetate inhibits metastatic breast cancer cell growth in vitro and in vivo. Breast Cancer Res Treat. 2010; 120(1): 253-60.

63. Papandreou I, Goliasova T, Denko NC. Anticancer drugs that target metabolism: Is dichloroacetate the new paradigm? Int J Cancer. 2011; 128(5): 1001-8.

64. Kushnareva Y, Murphy AN, Andreyev A. Complex I-mediated reactive oxygen species generation: modulation by cytochrome $\mathrm{c}$ and NAD(P)+ oxidation-reduction state. Biochem J. 2002; 368(Pt 2): 545-53.

65. Kinnaird A, Michelakis ED. Metabolic modulation of cancer: a new frontier with great translational potential. J Mol Med (Berl). 2015; 93(2): 127-42.

66. Sutendra G, Dromparis P, Kinnaird A, et al. Mitochondrial activation by inhibition of PDKII suppresses HIF1a signaling and angiogenesis in cancer. Oncogene. 2013; 32(13):1638-50.

67. Lin G, Hill DK, Andrejeva G, et al. Dichloroacetate induces autophagy in colorectal cancer cells and tumours. Br J Cancer. 2014; 111(2):375-85.

68. Michelakis ED, Sutendra G, Dromparis P, et al. Metabolic modulation of glioblastoma with dichloroacetate. Sci Transl Med. 2010; 2(31):31ra34.

69. Kaufmann P, Engelstad K, Wei Y, et al. Dichloroacetate causes toxic neuropathy in MELAS: a randomized, controlled clinical trial. Neurology. 2006; 66(3): 324-30.

70. Stacpoole PW, Gilbert LR, Neiberger RE, et al. Evaluation of long-term treatment of children with congenital lactic acidosis with dichloroacetate. Pediatrics. 2008; 121(5): e1223-8.

71. Shroads AL, Guo X, Dixit V, et al. Age-dependent kinetics and metabolism of dichloroacetate: possible relevance to toxicity. J Pharmacol Exp Ther. 2008; 324(3): 1163-71.

72. Cairns RA, Papandreou I, Sutphin PD, et al. Metabolic targeting of hypoxia and HIF1 in solid tumors can enhance cytotoxic chemotherapy. Proc Natl Acad Sci U S A. 2007; 104(22): 9445-50.

73. Ishiguro $\mathrm{T}$, Ishiguro $\mathrm{R}$, Ishiguro $\mathrm{M}$, et al. Co-treatment of dichloroacetate, omeprazole and tamoxifen exhibited synergistically antiproliferative effect on malignant tumors: in 
vivo experiments and a case report.

Hepatogastroenterology. 2012; 59(116): 994-6.

74. Xuan Y, Hur H, Ham IH, et al. Dichloroacetate attenuates hypoxia-induced resistance to 5 -fluorouracil in gastric cancer through the regulation of glucose metabolism. Exp Cell Res. 2014; 321(2): 219-30.

75. Tong J, Xie G, He J, et al. Synergistic antitumor effect of dichloroacetate in combination with 5-fluorouracil in colorectal cancer. J Biomed Biotechnol. 2011; 2011: 740564.

76. Pathak RK, Marrache S, Harn DA, et al. Mito-DCA: a mitochondria targeted molecular scaffold for efficacious delivery of metabolic modulator dichloroacetate. ACS Chem Biol. 2014; 9(5): 1178-87.

77. Dhar S, Lippard SJ. Mitaplatin, a potent fusion of cisplatin and the orphan drug dichloroacetate. Proc Natl Acad Sci U S A. 2009; 106(52): 22199-204.

78. Xie J, Wang BS, Yu DH, et al. Dichloroacetate shifts the metabolism from glycolysis to glucose oxidation and exhibits synergistic growth inhibition with cisplatin in HeLa cells. Int J Oncol. 2011; 38(2): 409-17.

79. Tso SC, Qi X, Gui WJ, et al. Structure-guided development of specific pyruvate dehydrogenase kinase inhibitors targeting the ATP-binding pocket. J Biol Chem. 2014; 289(7): 4432-43.

80. Meng T, Zhang D, Xie Z, et al. Discovery and optimization of 4,5-diarylisoxazoles as potent dual inhibitors of pyruvate dehydrogenase kinase and heat shock protein 90. J Med Chem. 2014; 57(23): 9832-43. 